\title{
Combining the Radon, Markov, and Stieltjes Transforms for Object Reconstruction
}

\author{
Annie Cuyt and Brigitte Verdonk \\ Dept of Mathematics and Computer Science, University of Antwerp \\ Middelheimlaan 1, B-2020 Antwerpen, Belgium \\ \{annie.cuyt, brigitte.verdonk\}@ua.ac.be
}

\begin{abstract}
In shape reconstruction, the celebrated Fourier slice theorem plays an essential role. By virtue of the relation between the Radon transform, the Fourier transform and the 2-dimensional inverse Fourier transform, the shape of an object can be reconstructed from the knowledge of the object's Radon transform. Unfortunately, a discrete implementation requires the use of interpolation techniques, such as in the filtered back projection.

We show how the need for interpolation can be overcome by using the relationship between the Radon transform, the Markov transform and the 2-dimensional Stieltjes transform. When combining the knowledge of an object's Radon transform for discrete angles $\theta$, with the less well-known Padé slice theorem, the object under consideration can be reconstructed from the solution of a linear least squares problem.
\end{abstract}

\section{The Radon, Markov, and Stieltjes Integral Transforms}

The Radon transform $R_{\vec{\xi}}(u)$ of a square-integrable $n$-variate function $f(\vec{x})$ with $\vec{x}=\left(x_{1}, \ldots, x_{n}\right)$ is defined as

$$
R_{\vec{\xi}}(u)=\int_{\mathbb{R}^{n}} f(\vec{x}) \delta(\vec{\xi} \vec{x}-u) d \vec{x} \quad d \vec{x}=d x_{1} \ldots d x_{n}
$$

with $\|\vec{\xi}\|=1$ and $\vec{\xi} \vec{x}=u$ an $(n-1)$-dimensional manifold orthogonal to $\vec{\xi}$. When $n=2, \vec{\xi}$ is fully determined by an angle $\theta$ and

$$
R_{\theta}(u)=\int_{-\infty}^{+\infty} \int_{-\infty}^{+\infty} f(t, s) \delta(t \cos \theta+s \sin \theta-u) d t d s
$$

In the sequel of the text, to simplify notation, we mainly focus on the twodimensional case, without loss of generality. Let the square-integrable function $f(t, s)$ be defined in a compact region $A$ of the first quadrant $t \geq 0, s \geq 0$ of the plane. According to a fundamental property of the Radon transform $R_{\theta}(u)$ of $f(t, s)[5]$, the following relation holds for any square-integrable function $F(u)$ :

$$
\int_{-\infty}^{+\infty} R_{\theta}(u) F(u) d u=\int_{0}^{\infty} \int_{0}^{\infty} f(t, s) F(t \cos \theta+s \sin \theta) d t d s
$$


If we take $F(u)=1 /(1+z u)$, then

$$
g_{\theta}(z)=\int_{-\infty}^{+\infty} \frac{R_{\theta}(u)}{1+z u} d u=\int_{0}^{\infty} \int_{0}^{\infty} \frac{f(t, s)}{1+(t \cos \theta+s \sin \theta) z} d t d s
$$

A Markov function is defined to be a function with an integral representation

$$
\left.\left.g(z)=\int_{a}^{b} \frac{f(u)}{1+z u} d u \quad-\infty<a \leq 0 \leq b<+\infty, z \notin\right]-\infty,-1 / b\right] \cup[-1 / a,+\infty[
$$

where $f(u)$ is non-trivial and positive and the moments

$$
c_{i}=\int_{a}^{b} u^{i} f(u) d u \quad i=0,1, \ldots
$$

are finite. If $f$ is nonzero in $[a, b]$ with $0<a<b$ then (B] is considered on $[0, b]$. If $f$ is nonzero in $[a, b]$ with $a<b<0$, then (3) is considered on [a,0]. A Markov series is defined to be a series

$$
\sum_{i=0}^{\infty}(-1)^{i} c_{i} z^{i}
$$

which is derived by a formal expansion of (3). The Markov function $g(z)$ is also called the Markov transform of the function $f(u)$. Furthermore, in case (5) is the formal series expansion of a Markov function with a nonzero radius of convergence, the Markov moment problem, in which one reconstructs $f(u)$ from the moments $c_{i}$, is determinate.

A bivariate Stieltjes function $g(z, w)$ is defined by the integral representation

$$
g(z, w)=\int_{0}^{\infty} \int_{0}^{\infty} \frac{f(t, s)}{1+(z t+w s)} d t d s
$$

where $f(t, s)$ is non-trivial and positive. Its finite real-valued moments are given by

$$
c_{i j}=\int_{0}^{\infty} \int_{0}^{\infty} t^{i} s^{j} f(t, s) d t d s
$$

A formal expansion of (6) provides a bivariate Stieltjes series

$$
\sum_{i, j=0}^{\infty}\left(\begin{array}{c}
i+j \\
i
\end{array}\right)(-1)^{i+j} c_{i j} z^{i} w^{j}
$$

The function $g(z, w)$ is also called the bivariate Stieltjes transform of $f(t, s)$.

Now let us have another look at (2) and identify our object under reconstruction with its characteristic function. If $f(t, s)$ is the characteristic function of a compact set $A$ lying in the first quadrant, then $g_{\theta}(z)$ is a Markov function, because $R_{\theta}(u)$ is zero outside a region of compact support. Furthermore, since $g_{\theta}(z)=g(z \cos \theta, z \sin \theta)$, there is a close link between the bivariate Stieltjes transform of the characteristic function of $A$ and the Markov transform of its Radon transform. In order to translate these properties into an algorithm for the reconstruction of $A$ from the knowledge of its Radon transform $R_{\theta}(u)$, we need to detail how its Markov transform can be computed. 


\section{Reconstruction Algorithm}

Let the unknown object $A$ which we identify with its characteristic function lie in the first quadrant and within the unit circle. This is a matter of shifting and scaling. The reconstruction of $A$ then goes as follows.

- Input of the algorithm is some indirect information that is available on the object $A$, namely its Radon transform for a discrete number of angles $\theta_{n}$ (bivariate case). If the univariate moments $C_{\ell}^{(\theta)}$ of the Radon transform or the multivariate moments $c_{i j}$ of $f(t, s)$ are given instead, one skips the first, respectively the first two steps of the algorithm.

- Compute the moments

$$
C_{\ell}^{(\theta)}=\int_{a(\theta)}^{b(\theta)} u^{\ell} R_{\theta}(u) d u
$$

for a discrete number of angles $\theta=\theta_{n}$ with $0 \leq n \leq N$. From the parameterized moments

$$
C_{\ell}^{\left(\theta_{n}\right)}=\sum_{i=0}^{\ell}\left(\begin{array}{l}
\ell \\
i
\end{array}\right) c_{i, \ell-i} \cos ^{i} \theta_{n} \sin ^{\ell-i} \theta_{n} \quad \ell=0,1,2, \ldots
$$

the bivariate moments $c_{i, \ell-i}$ can be computed by solving (8), possibly in the least

- With the moments $c_{i j}$ one computes, for successive $m$, the bivariate homogeneous Padé approximant 32] $r_{m-1, m}(z, w)$ of the Stieltjes transform $g(z, w)$. Increasing $m$ to $m+1$, implies adding the moments $c_{i, 2 m-i}$ and $c_{i, 2 m+1-i}$ to the data. It is well-known [1, p. 228] that on each slice $S_{\theta_{n}}$ the sequence $\left\{r_{m-1, m}(z)\right\}_{m \in \mathbb{N}}$ converges rapidly to $g(z, w)$ restricted to that slice. The relationship between $m$ and $N$ is $N=2 m+1$ with $N$ usually rather small.

- At the same time, for each $-\pi / 2<\theta_{n} \leq \pi / 2$ and each $0 \leq z_{j} \leq 1$, the value of the Stieltjes transform $g(z, w)$ evaluated at $\left(z_{j} \cos \theta_{n}, z_{j} \sin \theta_{n}\right)$ can be approximated to high accuracy by a cubature formula

$$
\sum_{i=1}^{L} \frac{\omega_{i}}{1+z_{j}\left(t_{i} \cos \theta_{n}+s_{i} \sin \theta_{n}\right)} f\left(t_{i}, s_{i}\right) \quad n=0,1, \ldots, \quad j=0,1, \ldots
$$

with weights $\omega_{i}$ and nodes $\left(t_{i}, s_{i}\right)$. Subsequently the values $f\left(t_{i}, s_{i}\right)$ are computed from the least squares problem

$$
\begin{aligned}
\sum_{i=1}^{L} \frac{\omega_{i}}{1+z_{j}\left(t_{i} \cos \theta_{n}+s_{i} \sin \theta_{n}\right)} f\left(t_{i}, s_{i}\right) & \approx g\left(z_{j} \cos \theta_{n}, z_{j} \sin \theta_{n}\right) \\
& =\lim _{m \rightarrow \infty} r_{m-1, m}\left(z_{j} \cos \theta_{n}, z_{j} \sin \theta_{n}\right)
\end{aligned}
$$

- The reconstruction of $A$ is identified with $A \approx\left\{\left(t_{i}, s_{i}\right) \mid f\left(t_{i}, s_{i}\right) \geq 0.5\right\}$ where the threshold 0.5 is chosen because for the original shape $f(t, s)=1$ inside $A$ and $f(t, s)=0$ outside $A$. 
Since the homogeneous Padé approximant can be defined analogously in any number of variables, the procedure for three-dimensional shape reconstruction is entirely similar.

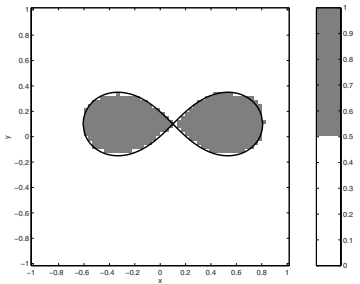

Fig. 1. $A=\left\{(t, u) \mid\left((t-0.1)^{2}+(u-\right.\right.$ $\left.0.1)^{2}+1 / 4^{2}-(t-0.1)^{2}=1 / 16\right\} \# \theta_{n}=$ $80, \# z_{j}=60, h=k=1 / 16, m=10, \epsilon=$ $5.3 \times 10^{-7}$

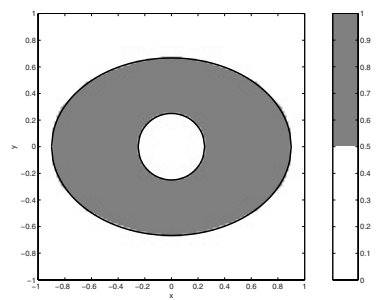

Fig. 2. $A=\left\{(t, u) \mid 81 t^{2} / 100+4 u^{2} / 9 \leq\right.$

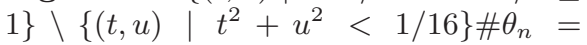
$25, \# z_{j}=15, h=k=1 / 32, m=10, \epsilon=$ $1.2 \times 10^{-4}$

Within the set of interesting objects $A$ we present a non-convex example (Figure 1) and an example with non-connected boundary (Figure 2). We delimit the original shape in black, show the reconstructed area in grey and list the number of angles $\theta_{n}$ and the number of radial points $z_{j}$ used in the least squares formulation (9), the degree $m$ of the Padé denominator and the relative error $\epsilon=\max _{x^{2}+y^{2} \leq 1}\left|r_{m-2, m-1}-r_{m-1, m}\right| /\left|r_{m-1, m}\right|$ in the computation of the Padé approximant. The value $\epsilon$ is an estimate of the relative error present in the right hand side of the linear least squares problem (9). The least squares problem (9), which is an inverse problem, is in general ill-conditioned and therefore a regularization technique must be applied. In all of the following examples we have found the technique known as truncated SVD [4] to do an excellent job.

For the approximation of $g\left(z_{j} \cos \theta_{n}, z_{j} \sin \theta_{n}\right)$ we use the simple compound 4point Gauss-Legendre product rule [6, pp. 230-231] with $h=1 / 16=k$ for Figure 1 and $h=1 / 32=k$ for Figure 2 .

\section{References}

1. G.A. Baker, Jr. and P. Graves-Morris. Padé approximants (2nd Ed.). Cambridge University Press, 1996.

2. A. Cuyt. A comparison of some multivariate Padé approximants. SIAM J. Math. Anal., 14:195-202, 1983.

3. A. Cuyt. Padé approximants for operators: theory and applications. LNM 1065, Springer Verlag, Berlin, 1984.

4. P.C. Hansen. The truncated SVD as a method for regularization. BIT, 27:543-553, 1987.

5. A.C. Kak and M. Slaney. Principles of Computerized Tomographic Imaging. IEEE Press, 1988.

6. A.H. Stroud. Approximate calculation of multiple integrals. Prentice-Hall, 1971. 